\title{
New Architecture for Corporate Integration of Simulation and Production Control in Industrial Applications
}

\author{
E. Freund, A. Hypki, D. Pensky \\ Institute of Robotics Research (IRF), University of Dortmund \\ Otto-Hahn-Str. 8 \\ D-44227 Dortmund, Germany \\ email: \{freund, hypki, pensky\}@irf.de
}

\begin{abstract}
These days systems for 3D simulation and flexible control of robot-based workcells are in common use. However especially small and midsize companies do not make use of the advantages these technologies imply. Reasons for this behavior are the costs of the soft- and hardware systems as well as the fact that you need experts to operate those systems. This article describes an innovative architecture that has been developed for a corporate integration of simulation and control systems into production processes. The coexistence and cooperation of PC-based simulation and control makes the cost-effective use of Virtual Reality (VR) methods and a wide range of novel industrial applications possible. By use of VR technologies complex systems can be operated by any user without long periods of training. Thus, small and midsize companies are enabled to use innovative technologies to increase their productivity and competitiveness.
\end{abstract}

\section{Introduction}

As a result of increasingly shorter development cycles for new products there is a growing demand for production systems to offer highest possible product flexibility. It is necessary to integrate new products without impairing the current production. Moreover simulation and control systems are needed, which permit a close-to-reality simulation in connection with the possibility to transfer the tested programs into real operation as fast as possible.

All through the development of simulation and control systems for industrial production the requirements of small and midsize companies have not been respected. These companies are not able to spend time and money for installing complex and difficult-to-use systems. Because of the lack of high-qualified employees software systems have to become more and more easy to use in general. PC- and Windows ${ }^{\mathrm{TM}}$-based systems are suited concerning their well-known user interfaces and low priced hardware.

The Institute of Robotics Research (IRF), Dortmund, Germany and its partners develop systems for the whole range of modern automation technology. The systems include planning and optimization of the layout, the programming of the automation components including the upper control hierarchies, the simulation of the entire workcell up to control and teleservice of the real cell.

The PC-based 3D simulation system COSIMIR ${ }^{\circledR}$ (Cell Oriented Simulation of Industrial Robots) and the flexible workcell controller LUCAS (Layered Universal Controller for Automation Systems) have been integrated into an innovative architecture to meet the needs especially of small and midsize companies.

The new type of architecture is described on the basis of two research projects.

Within the scope of the project DEMON a novel system structure has been developed for disassembly tasks executed by a robot-based workcell. Virtual Reality techniques make the operation of the control system easier.

The project FA-SiCo (Factory Automation - Simulation and Control) includes the development of methods for a so-called Parallel Simulation of production processes. Using this technology opens up new applications for corporately integrated simulation and control systems. 


\section{Architecture}

In Figure 1 the structure of the integration of VR, simulation, and workcell control is presented. The left side of Figure 1 shows the different tasks realized by the usage of the workcell simulation system COSIMIR ${ }^{\circledR}$. The 3D-simulation system COSIMIR ${ }^{\circledR}$, which supports the simulation of entire workcells [1], was developed at the IRF [2]. It is PC-based and is running under Microsoft Windows ${ }^{\mathrm{TM}}$. Due to its ergonomic user interface supporting the complete range of Windows standards the training periods are minimized.

The whole process starts with the planning of the workcell layout, i. e. the generation of a model for further programming and simulation operations. This modeling is accomplished with the help of libraries, including for example industrial robots, PLCs, and mechanisms like conveyor belts and grippers. The 3D model of the workcell is used for the visualization within the Virtual Reality environment, being displayed for example in a head mounted display. The user selects the necessary tools and executes the desired tasks. These actions are interpreted by the VR system and are transferred into robot programs. These programs are tested in the simulation environment of COSIMIR ${ }^{\circledR}$, especially taking care of aspects like the reachability of all positions and of movements without collisions.

The right area of Figure 1 shows the different tasks of the flexible workcell controller LUCAS [3]. During configuration time the setup, e. g. of the communication channel (TCP/IP, ProfiBus, RS232 etc.), for each single automation component integrated in the whole workcell is done. Afterwards the coordination of the different devices is programmed using the process plan language implemented in LUCAS.

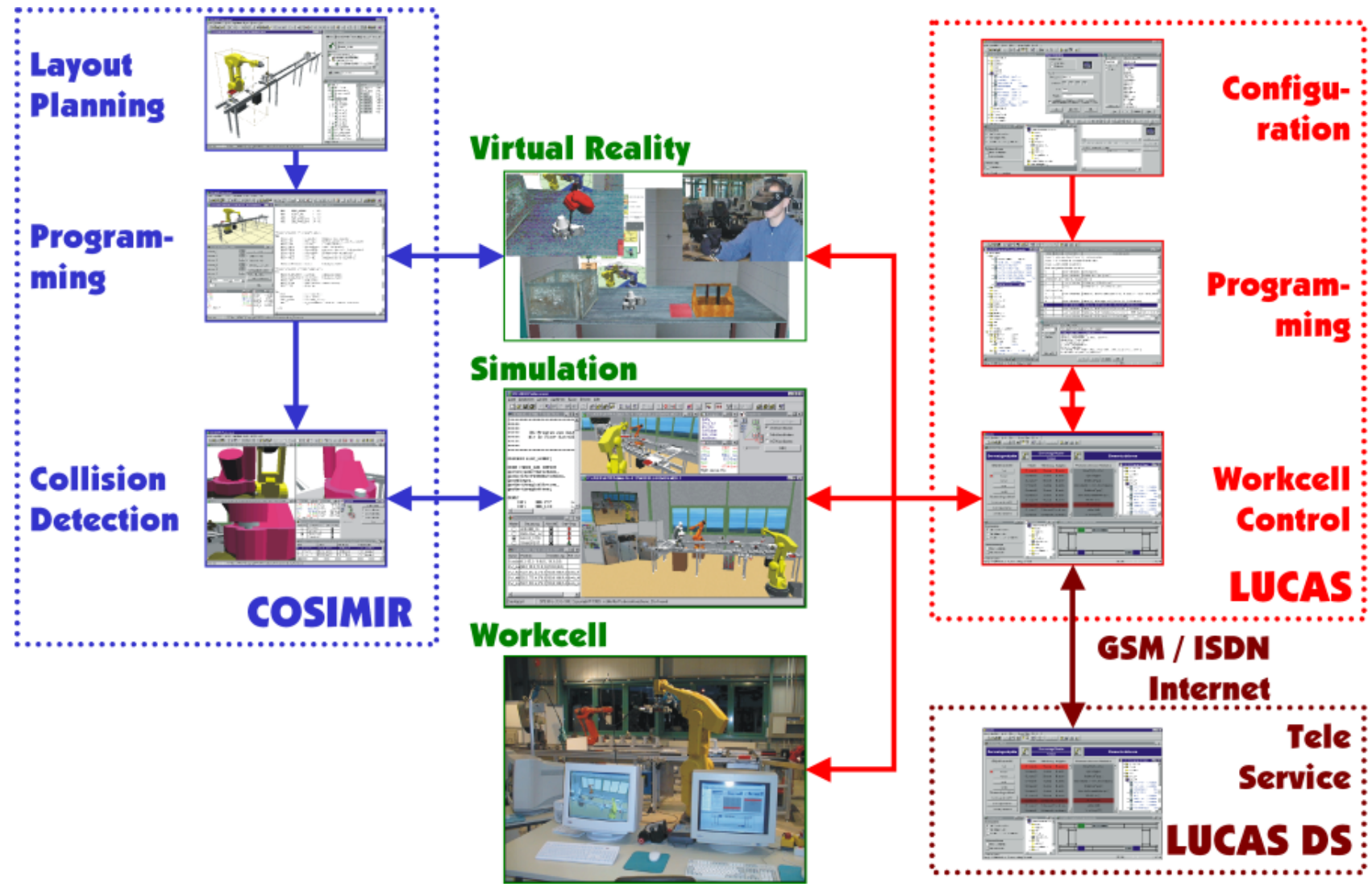

Figure 1: Connection Between Simulation and Control System 
These steps create the basis for the first integration of simulation and workcell control, i.e. also the first interaction of COSIMIR $^{\circledR}$ and LUCAS. LUCAS is responsible for loading the programs, developed and tested using COSIMIR $^{\circledR}$ as described before, into the different simulated automation devices. Additionally LUCAS is also responsible for the control of the whole workcell environment. The behavior of the complete workcell is simulated. In addition to the simulation of single robot programs done, aspects like material flow, coordination of the devices, and the correctness of the sequences of the different processing steps are also simulated. By this integration of simulation and control, based on exactly those programs used for the real workcell later on, a very high transferability of the achieved results is guaranteed.

After successful tests within the simulation environment LUCAS switches between the simulated and the real world by downloading the programs for the robots, PLCs, etc. into the physical automation devices. Thus LUCAS takes control of the complete workcell.

During production time new robot programs may be created and simulated at any time and then transferred into the real workcell without stopping the operation of the workcell. The teleservice components of LUCAS are based on different communication layers like mobile phones, ISDN, and Internet. They support the monitoring of actual states of the workcell as well the possibility to modify programs from any location.

\section{Project DEMON}

Due to the rising necessity of recycling as a base for saving raw materials and avoiding garbage the area of disassembly becomes increasingly interesting and opens new challenges in automation. All objects at the end of the life cycle have changed their outer appearance because of external influences as well as of ageing. This results in unique objects, each one to be handled in a different manner.

The main goal of the project DEMON was to design and build up demonstrators for a new control structure to satisfy the demands of automated disassembling. The objects to be disassembled were selected from automotive industry; one task was to unscrew car wheels, the other example was the disassembly of fuel pumps.

Figure 2 shows the robot unscrewing a car wheel. The model for the simulation and the VR environment is automatically generated and permanently updated according to sensor information. The robot programs are created using this model, too e.g. to define the position of each single screw.

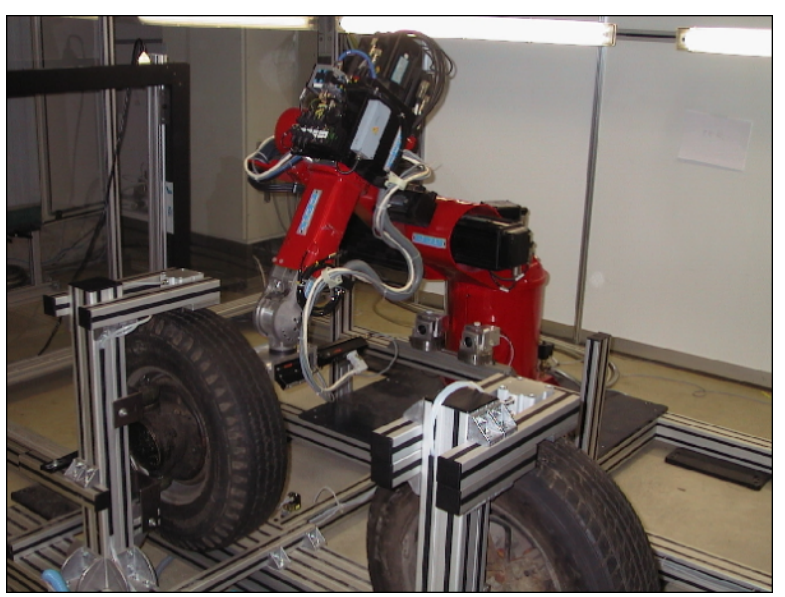

Figure 2: Workcell to unscrew car wheels

The workcell for automated fuel pump disassembly is build up of 3 robots as shown in Figure 3. The first robot inspects the pumps with a camera system to determine the number and the location of the screws, robot 2 polishes the screws to avoid sticking to the screwing tool mounted on the third robot.

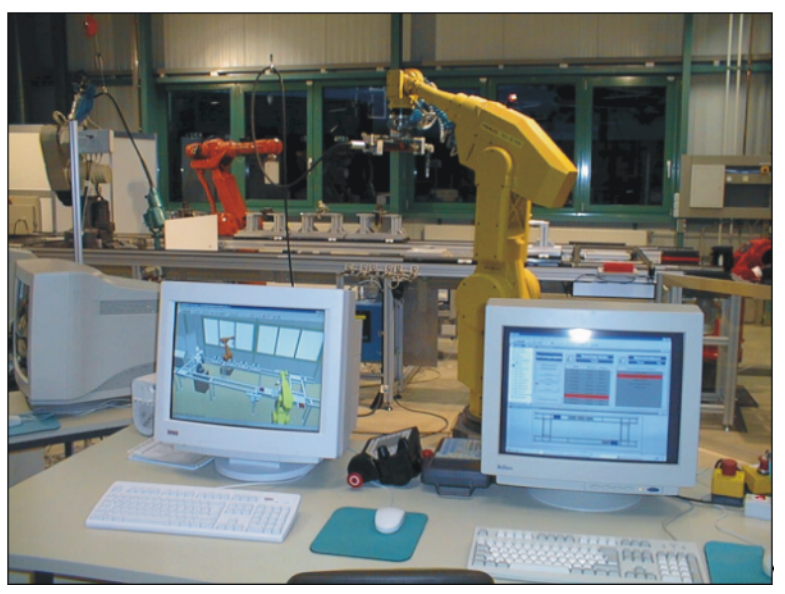

Figure 3: Disassembling of fuel pumps

Robot 3 is equipped with a gripper exchange system to hold the screwing tool as well as a gripper to handle the parts of the pump and to sort them according to their material. Figure 3 also presents the user interfaces of COSIMIR $^{\circledR}$ on the left and LUCAS on the right monitor. 


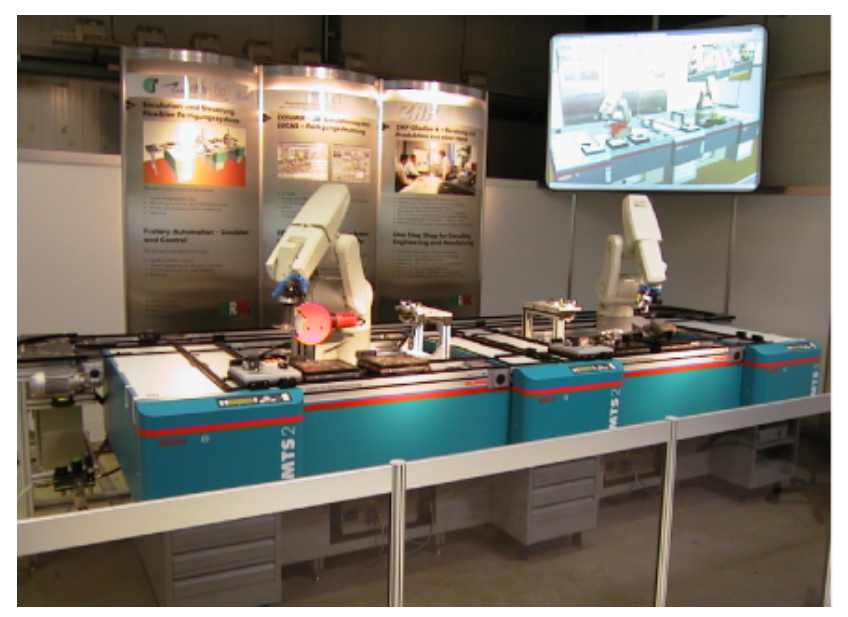

Reality

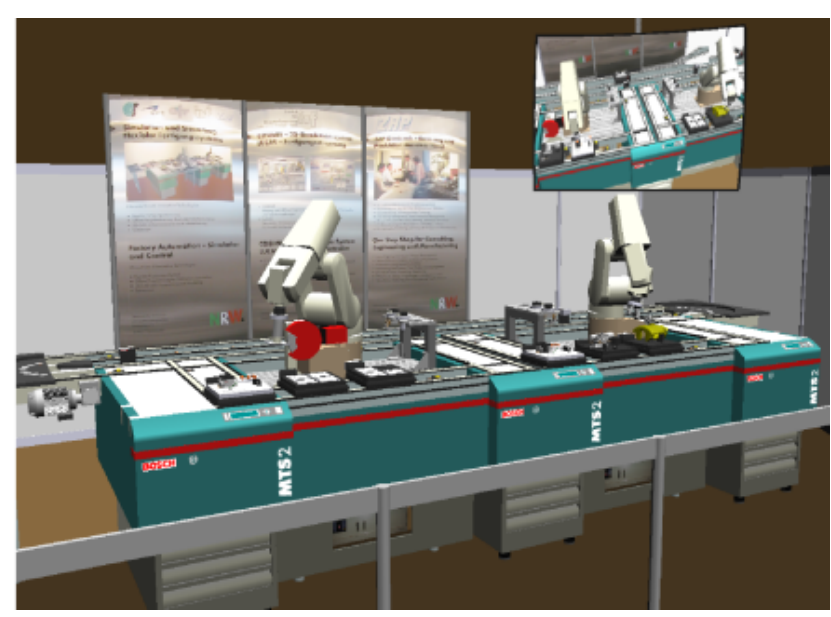

Simulation

\section{Figure 4: Close-to-Reality Simulation of the FA-SiCo Demonstrator with COSIMIR ${ }^{\circledast}$}

\section{Project FA-SiCo}

The goal of the project Factory Automation - Simulation and Control (FA-SiCo) was to demonstrate that by the use of innovative technologies especially small and midsize companies are able to meet new requirements because of decreasing development cycles of products. Therefore a fabrication system was built up to explain the efficient and cost-effective application of these technologies.

The manufacturing system shown in Figure 4 and consisting of a transfer system and two robots provides highest product flexibility as well as an intuitive user interface and optimized use of the integrated components. Several systems are integrated into the demonstrator's software including the modeling of the automation components and products, the simulation of all production processes, the workcell control, and teleservice.

\section{Close-to-Reality Simulation}

The 3D simulation system COSIMIR ${ }^{\circledR}$ is used for a closeto-reality simulation and the programming of the automation components within the scope of the project FA-SiCo (see Figure 4). For modeling of the demonstrator's simulation model existing model libraries were used. The product parts were created with a 3DCAD system and they were imported into the simulation model after being converted to one of the graphic exchange formats supported by COSIMIR ${ }^{\circledR}$. By the use of the simulation model the positions of the robots and the gripper exchange systems were determined relatively to the other workcell components.

The 3D simulation of robot-based workcells by COSIMIR $^{\circledR}$ includes simulation of robot programs, PLCs, sensors, actuators, material flow, and simulation of handling and treatment processes.

All programs for the two Mitsubishi RV-E4NM robots are entirely developed within $\operatorname{COSIMIR}^{\circledR}$ using the programming language MELFA Basic. The download and upload of programs and position lists is performed via an RS-232 interface. Because of small differences between simulation model and reality, commonly different position lists for the simulated and the real robots are used.

\section{Hierarchical Control}

During the FA-SiCo project LUCAS was used for coordination of the automation components. Via communication interfaces to the robots (RS-232) and to the material flow system (CANOpen) the production controller starts process tasks in the components at the right time using a predefined process plan for each product. All production data is stored in a standard database and can be accessed by overlaid production planning and control systems. The integrated process visualization is used to provide an easy and intuitively to use interface to monitor and to operate all production processes. 


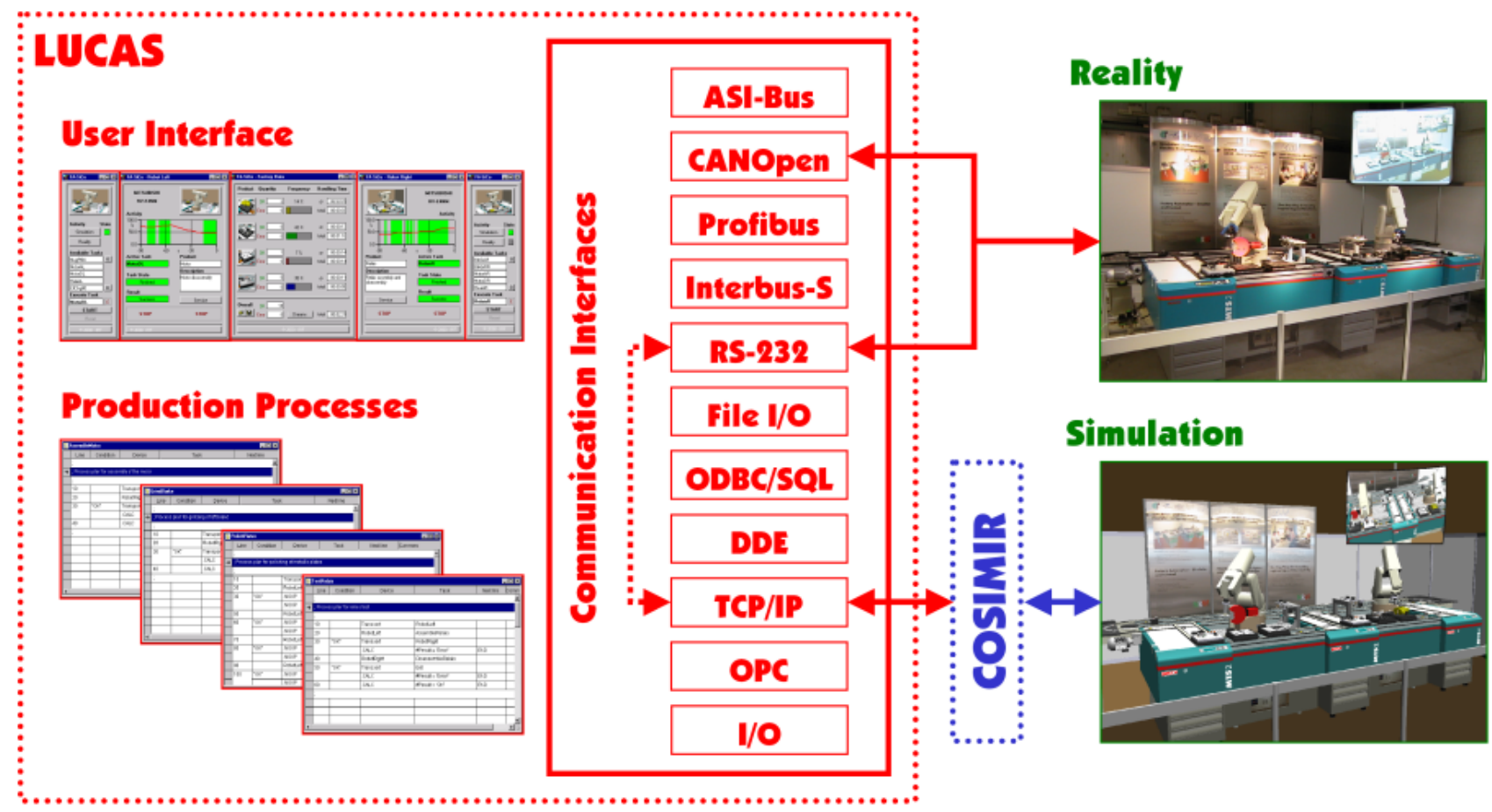

Figure 5: Connection Between Simulation and Control System for Parallel Simulation

The DS (Distributed System) functionality of LUCAS is used to integrate teleservice techniques into the controller system. An appropriate connection between the workcell controller and the teleservice station has to be established. Because the system uses standard network functionality, communication media like LAN or Internet via analog modem, GSM connection, or ISDN are suitable.

\section{Control meets Simulation}

For optimization of production processes a powerful connection between simulation and control system is mandatory. Thereby all overlaid programmable coordination procedures can be developed using the simulation. For that purpose the real components are not needed.

The systems COSIMIR ${ }^{\circledR}$ and LUCAS can be connected via a network based interface. Because it is based on TCP/IP the connection can be established over an ordinary Internet connection.

The different process tasks commonly being sent to the real components are executed by the simulated components. Using the close-to-reality simulation of
COSIMIR $^{\circledR}$ cycle times for production processes can be determined and optimized while executing the overlaid coordination processes by LUCAS. Moreover operators can be trained with the simulation using the same graphical user interface as for operating the real workcell. Figure 5 illustrates the described connection for the FA-SiCo demonstrator.

A wide range of industrial applications is supported by this powerful link between simulation and control methods:

- Offline-Programming of Control Structures, Batch Processes and Development of Graphical Operator Interfaces

- Operator Training and VR Operation

- Simulation in Advance

- 3D Process Visualization

While actual production is running simulation can be carried out in parallel. The parallel simulation is possible because of the controller's structure. Every process task can be sent to both simulation and reality. Thus, the connection of simulation and control system also provides a $3 \mathrm{D}$ visualization of production processes. 


\section{Summary}

This paper presents a new approach to integrate workcell simulation and workcell control in combination with Virtual Reality components to facilitate workcell programming. New structures like the one described are necessary to meet the requirements of industrial automation processes. Especially in the field of automated disassembly the conventional robot programming by Teach-In does not lead to satisfying results.

The integrated system is based on the 3D simulation system COSIMIR $^{\circledR}$ and the flexible workcell controller LUCAS. The Virtual Reality extensions for COSIMIR ${ }^{\circledR}$ are used as interface to the head mounted display and to the data glove on the one side. On the other side these extension modules fulfill the interpretation of user action and they are responsible for the automated generation of robot programs.

The VR and simulation system COSIMIR ${ }^{\circledR}$ is connected to the workcell controller LUCAS. By this integration of simulation and control, based on exactly those programs used for the real workcell later on, a very high transferability of the achieved results is guaranteed.

According to the developed structure three different workcells were built up to transfer the theoretical approach into practical environments. These realizations have proven the advantages of the concept and have shown a significant reduction in programming time. During operation these systems guarantee a reduction of faults combined with an increase in productivity.

\section{Acknowledgement}

Funding for this work was provided by the German Federal Ministry of Education and Research (BMBF) and the Ministry of Economics and Small Businesses, Technology and Transport (MWMTV) for North RhineWestphalia, Germany.

\section{References}

[1] Freund, E.; Hypki, A.; Uthoff, J. (1996). COSIMIR: Simulation of Complete Robotic Workcells. Proceedings of the 12th International Conference on CAD/CAM Robotics and Factories of the Future, London

[2] Freund, E.; Hypki, A.; Uthoff, J. (1997). New Strategies in Programming and Control of Flexible Manufacturing and Automated Disassembling Processes. Proceedings of the 13th International Conference on CAD/CAM Robotics and Factories of the Future, Pereira

[3] Freund, E.; Rothert, B.; Theis, K.; Uthoff, J. (1996). Comprehensive Approach to Control of Flexible Manufacturing Systems. Proceedings of the 12th International Conference on CAD/CAM Robotics and Factories of the Future, London 